\title{
Systolic blood pressure amplification and waveform calibration
}

\author{
Hypertension Research (2017) 40, 518; doi:10.1038/hr.2016.181; published online 19 January 2017
}

With great interest we read the paper by Nakagomi et al. ${ }^{1}$ recently published in the journal. We want to congratulate the authors and also take the opportunity to point out some additional aspects of their important work. Nakagomi et al. ${ }^{1}$ independently confirmed earlier work $^{2}$ showing that different methods to calibrate a peripheral artery waveform ( $\mathrm{C} 1$ based on systolic/diastolic pressure and C2 based on mean/diastolic pressure) influence the absolute accuracy of both the aortic pressure estimation as well as the 'absolute' systolic pressure amplification measured in millimeters of mercury (SBPA). These observations are in alignment with other investigators showing that calibration mode significantly influences associations to clinical outcomes. ${ }^{3,4}$

Non-invasively assessed aortic to peripheral pulse wave amplification is intentionally a 'relative' measure that is primarily pressure and calibration independent and driven by the peripheral pulse wave shape and the applied generalized transfer function alone. A major problem of estimating 'absolute' central blood pressure (BP) based on any kind of generalized transfer function is the use of inaccurate brachial cuff measured BP as a calibration standard. ${ }^{5}$ This is illustrated by the observations by Nakagomi et al., ${ }^{1}$ that SBPA did not differ for both calibration methods (C1 and C2), when invasively recorded pressures were used for calibration.

These data provide the intriguing possibility to accurately calculate the 'true' (invasive) brachial systolic BP. We suggest this may be achieved based on the 'non-invasively' estimated aortic pressure derived from C2 together with the 'relative SBPA' derived from C1. Although not explicitly discussed in their paper, the authors report C1 based SBPA being 1.09 (141.9 $\mathrm{mm} \mathrm{Hg} / 130.0 \mathrm{~mm} \mathrm{Hg}-$ derived from Table 2). Multiplying C2 based non-invasive aortic systolic pressure (again from Table 2) with C1 based SBPA, we calculate $149.5 \mathrm{~mm} \mathrm{Hg} \times 1.09=162.95 \mathrm{~mm} \mathrm{Hg}$ as an estimate for the 'true' invasive brachial systolic pressure. Indeed, Nakagomi et al. ${ }^{1}$ report the invasive brachial systolic $\mathrm{BP}$ to be $164.1 \mathrm{~mm} \mathrm{Hg}$, which is close to our estimate. It would be interesting if the authors could confirm this analysis based on their original data.

At a first glance this mixture of three methods seems to be both amazing as well as confusing. A closer look unveils some major issues to be considered. First, brachial systolic and diastolic pressures actually used in clinical routine relate to a different reference standard (Korotkoff) than invasive pressures (catheter) and this leads to inherent methodological offsets. The British Hypertension Society therefore explicitly omitted the invasive comparisons for oscillometric devices in their validation protocol. ${ }^{6}$ Second, although oscillometric mean arterial pressure measurement is device dependent and not standardized, only the oscillometric ${ }^{2,7}$ and the catheter, but not the the Korotkoff method, is capable to directly measure arterial mean pressure. Again potential method-driven offsets in pressure readings must be taken into account. Third, the result of a generalized transfer function is a priori invariant to pressure and calibration. Applying different pressure measurement paradigms to a given generalized transfer function will lead to results being consistent within each particular paradigm, but will be misleading if methods are mixed without considering their potential offsets and limitations.

Considering all the points raised, carefully designed and validated oscillometric approaches may be used to bridge the gap between Korotkoff and catheter readings. This appears to be demonstrated in the data provided by Nakagomi et al., ${ }^{1}$ and could have major implications towards improving the accuracy of non-invasive cuff BP methods.

\section{CONFLICT OF INTEREST}

The authors declare no conflict of interest.

Siegfried Wassertheurer ${ }^{1}$, Bernhard Hametner ${ }^{1}$, James Sharman ${ }^{2}$ and Thomas Weber ${ }^{3}$

${ }^{1}$ Health and Environment Department, AIT Austrian Institute of Technology, Vienna, Austria; ${ }^{2}$ Menzies Institute for Medical Research, University of Tasmania, Tasmania, Australia and ${ }^{3}$ Cardiology Department, Klinikum Wels-Grieskirchen, Wels, Austria E-mail: Siegfried.Wassertheurer@ait.ac.at

1 Nakagomi A, Okada S, Shoji T, Kobayashi Y. Comparison of invasive and brachial cuff-based noninvasive measurements for the assessment of blood pressure amplification. Hypertens Res 2017; 40: 237-242.

2 Weber T, Wassertheurer S, Rammer M, Maurer E, Hametner B, Mayer CC, Kropf J, Eber B. Validation of a brachial cuff-based method for estimating central systolic blood pressure. Hypertension 2011; 58: 825-832.

3 Wassertheurer S, Baumann M. Assessment of systolic aortic pressure and its association to all cause mortality critically depends on waveform calibration. J Hypertens 2015; 33: 1884-1888.

4 Negishi K, Yang H, Wang Y, Nolan MT, Negishi T, Pathan F, Marwick TH, Sharman JE. Importance of calibration method in central blood pressure for cardiac structural abnormalities. Am J Hypertens 2016; 29: 1070-1076.

5 Fagher B, Magnusson J, Thulin T. Direct and indirect blood pressure in normotensive and hypertensive subjects. Intern Med 1994; 236: 85-90.

6 O'Brien E, Atkins N. A comparison of the British Hypertension Society and Association for the Advancement of Medical Instrumentation protocols for validating blood pressure measuring devices: can the two be reconciled? J Hypertens 1994; 12: 1089-1094.

7 Ursino M, Cristalli C. A mathematical study of some biomechanical factors ffecting the oscillometric blood pressure measurement. IEEE Trans Biomed Eng 1996; 43: 761-778. 